\title{
Advection-dominated accretion flows with causal viscosity (Research Note)
}

\author{
R. Takahashi \\ Graduate School of Arts and Sciences, University of Tokyo, 153-8902 Tokyo, Japan \\ e-mail: rohta@ea.c.u-tokyo.ac.jp \\ Received 7 August 2006 / Accepted 2 October 2006 \\ ABSTRACT

\begin{abstract}
Aims. We present the basic equations and sample solutions for the steady-state global transonic solutions of the advection-dominated formula for obtaining the solutions of ADAFs are also presented.

Methods. We solve the transonic solutions of ADAFs by using the explicit numerical integrations, as in the Runge-Kutta method. In this calculation method, we first solve the physical values at the sonic radius where L'Hopital's rule is used. Then, we numerically the inward and outward directions.

Results. By the calculation procedures presented in this paper, we can cover all parameter spaces of the transonic solutions of ADAFs. Sample transonic solutions for ADAF-thick disk and ADAF-thin disk are presented. The explicit formula for the analytical expansion around the singular points, the sonic and the viscous points, are presented. If we set the diffusion timescale to null, the formalism in
\end{abstract} \\ accretion flows (ADAFs) with a causal viscosity prescription. The procedures for the stable numerical calculations and all explicit \\ solve the coupled differential equations of the radial velocity, the angular momentum, and the sound speed from the sonic radius in \\ this study becomes the formalism of the acausal viscosity that is usually used in past studies for calculating of the ADAF structure.
}

Key words. accretion, accretion disks - black hole physics - hydrodynamics

\section{Introduction}

Advection-dominated accretion flows (ADAFs) are one of the fundamental solutions for the accretion flows into the black holes in low-luminous systems, such as low-luminosity active galactic nuclei. In past studies, several methods have been used to obtain the transonic solutions of ADAFs, e.g., the relaxation scheme (e.g. Narayan et al. 1997), the implicit integration scheme (e.g. Kato et al. 1998), the outward integration scheme with the inner boundary condition (Becker \& Le 2003), or the inward or outward integration scheme with the special condition at and inside the sonic radius (Lu et al. 1999). All these works assume the diffusive viscosity with an infinite diffusive velocity.

In this paper, we give the method for solving the global transonic solutions of ADAFs with causal viscosity prescriptions that assume the finite diffusive velocity proposed by Papaloizou \& Szuszkiewicz (1994), which can become the alternative method for solving the global solutions. Gammie \& Popham (1998) calculate the ADAF structure in the Kerr metric with the causal viscosity prescription by using the relaxation scheme. In the calculations with the causal viscosity prescription, the boundary condition at the viscous point is used, so the boundary condition at the horizon is not required (see, also Sect. 5). If we set the diffusion timescale to null, the basic equations in this study become the basic equations for the case of the acausal viscosity, which is usually used in past studies.

After we present the basic equations for the ADAF with the causal viscosity prescription in Sect. 2 , the boundary conditions for the global transonic solutions of ADAFs with the causal viscosity are given in Sect. 3. The numerical procedures of this method of solving the transonic solutions for ADAFs and the sample solutions are presented in Sect. 4. Discussion and conclusions are given in Sects. 5 and 6, respectively.

\section{Basic equations of ADAF with causal viscosity}

The basic equations used in this paper are same as those in Narayan et al. (1997), except that we use the causal viscosity prescription. The mass conservation is written as

$-4 \pi r H \rho v_{\mathrm{r}}=\dot{M}$,

where $H$ is the half thickness of the accretion disk, $\rho$ the gas density, $v_{\mathrm{r}}$ the radial velocity, and $\dot{M}$ is the mass accretion rate. Here, we assume $v_{\mathrm{r}}<0$ and $H=(5 / 2)^{1 / 2} a_{\mathrm{s}} / \Omega_{\mathrm{K}}$ where $a_{\mathrm{S}}$ is the sound speed defined as $a_{\mathrm{s}}=(p / \rho)^{1 / 2}$ and $p$ is the pressure.

The radial momentum conservation is described as

$v_{\mathrm{r}} \frac{\mathrm{d} v_{\mathrm{r}}}{\mathrm{d} r}=\left(\Omega^{2}-\Omega_{\mathrm{K}}^{2}\right) r-\frac{1}{\rho} \frac{\mathrm{d} p}{\mathrm{~d} r}$,

where $\Omega$ is the angular velocity of the accretion flow and $\Omega_{\mathrm{K}}$ the Keplerian angular velocity calculated as $\Omega_{\mathrm{K}}=1 /\left[r^{1 / 2}(r-2)\right]$. Here, we set $G M=1$ and use the Paczyńskii-Wiita potential.

The energy equation is written as

$\frac{\rho v_{\mathrm{r}}}{\gamma-1} \frac{\mathrm{d} a_{\mathrm{s}}^{2}}{\mathrm{~d} r}-a_{\mathrm{s}}^{2} v_{\mathrm{r}} \frac{\mathrm{d} \rho}{\mathrm{d} r}=\rho v_{\mathrm{r}}(\ell-j) \frac{\mathrm{d} \Omega}{\mathrm{d} r}$,

where $\gamma$ is the ratio of specific heats of the accreting gas, $\ell\left(=\Omega r^{2}\right)$ the angular momentum, and $j$ the integration constant meaning the specific angular momentum at some radius.

For the case of the diffusive viscosity with the infinite diffusion velocity, the shear stress $t_{r \phi}$ is written as

$t_{r \phi}=-v \rho r^{2} \frac{\mathrm{d} \Omega}{\mathrm{d} r}$ 
Then, the angular momentum equation for the case of the acausal viscosity is written as

$\frac{\mathrm{d} \Omega}{\mathrm{d} r}=\frac{v_{\mathrm{r}}(\ell-j)}{v r^{2}}$,

where $v$ is the kinematic coefficient of viscosity, and, here $j$ is the specific angular momentum per unit mass where $\mathrm{d} \Omega / \mathrm{d} r=0$, if such a point exists. By assuming the $\alpha$-viscosity, we set $v=$ $\alpha a_{\mathrm{s}}^{2} / \Omega_{\mathrm{K}}$. From Eq. (5), the equation for the angular momentum is written as

$\frac{\mathrm{d} \ell}{\mathrm{d} r}=\frac{2 \ell}{r}+\frac{v_{\mathrm{r}}(\ell-j)}{v}$.

In the same way as Papaloizou \& Szuszkiewicz (1994) and Gammie \& Popham (1998), we include the effects of the diffusive viscosity with the finite diffusion velocity. By using the causal viscosity, we can derive the angular momentum equation with the diffusive viscosity, with the diffusion velocity $a_{v}$ written as

$\frac{\mathrm{d} \ell}{\mathrm{d} r}=\frac{\mathcal{N}_{v}}{\mathcal{D}_{v}}$

where

$\mathcal{D}_{v}=1-v_{\mathrm{r}}^{2} / a_{v}^{2}, \quad \mathcal{N}_{v}=\frac{2 \ell}{r}+\frac{v_{\mathrm{r}}(\ell-j)}{v}\left(1-\frac{2 v_{\mathrm{r}} \tau_{v}}{r}\right)$.

Here, $\tau_{v}$ is the relaxation timescale. For simplicity, we set $\tau_{v}$ and $a_{v}$ as $\tau_{v}=\Omega_{\mathrm{K}}^{-1}$ and $a_{v}=\left(v / \tau_{v}\right)^{1 / 2}$, respectively. Now, the shear stress for the causal viscosity is written as

$t_{r \phi}=\frac{-v \rho}{1-2 v_{\mathrm{r}} \tau_{v} / r}\left[\left(1-\frac{v_{\mathrm{r}}^{2}}{a_{v}^{2}}\right) r^{2} \frac{\mathrm{d} \Omega}{\mathrm{d} r}-\frac{2 \ell}{r} \frac{v_{\mathrm{r}}^{2}}{a_{v}^{2}}\right]$.

When $\tau_{v}=0$, i.e., $a_{v}=\infty$, Eqs. (7) and (9) for the causal viscosity become the equations for the acausal viscosity, Eqs. (6) and (4). Even as the point where $\mathrm{d} \Omega / \mathrm{d} r=0$, the shear stress $t_{r \phi}$ in general does not become null due to the effects of the causal viscosity. Since the effects of the causal viscosity are coupled with the radial velocity as $v_{\mathrm{r}}^{2} / a_{\gamma}^{2}$ and $v_{\mathrm{r}} \tau_{v}$ in Eqs. (7) and (9), the effects of the causal viscosity is effective in the inner region of the accretion flow where the absolute value of the radial velocity is large.

\section{Boundary conditions and physical values at the sonic radius}

Since the accretion flows plunge into the black hole supersonically after a sonic transition, the equations are singular at the sonic radius. In order to see this, from Eqs. (1)-(3), we can derive the equation for $\mathrm{d} v_{\mathrm{r}} / \mathrm{d} r$ as

$\frac{\mathrm{d} v_{r}}{\mathrm{~d} r}=\frac{\mathcal{N}}{\mathcal{D}}$

where

$$
\begin{aligned}
\mathcal{D}= & v_{\mathrm{r}}-\left(\frac{2 \gamma}{\gamma+1}\right) \frac{a_{\mathrm{s}}^{2}}{v_{\mathrm{r}}}, \\
\mathcal{N}= & \frac{\Omega^{2}-\Omega_{\mathrm{K}}^{2}}{r}-\left(\frac{2 \gamma}{\gamma+1}\right) a_{\mathrm{s}}^{2} \frac{\mathrm{d} \ln \left(\Omega_{\mathrm{K}} / r\right)}{\mathrm{d} r} \\
& -\left(\frac{\gamma-1}{\gamma+1}\right) \frac{(\ell-j)}{r^{2}}\left(\frac{\mathrm{d} \ell}{\mathrm{d} r}-\frac{2 \ell}{r}\right) .
\end{aligned}
$$

Here, Eq. (7) is inserted into $\mathrm{d} \ell / \mathrm{d} r$ and $\mathrm{d} \ln \left(\Omega_{\mathrm{K}} / r\right) / \mathrm{d} r=$ $-(5 r-6) /[2 r(r-2)]$. On the other hand, the equation for $\mathrm{d} a_{\mathrm{s}} / \mathrm{d} r$ is written as

$\frac{\mathrm{d} a_{\mathrm{s}}}{\mathrm{d} r}=\left(\frac{\gamma-1}{\gamma+1}\right)\left[-\frac{a_{\mathrm{s}}}{v_{\mathrm{r}}} \frac{\mathrm{d} v_{\mathrm{r}}}{\mathrm{d} r}+a_{\mathrm{s}} \frac{\mathrm{d} \ln \left(\Omega_{\mathrm{K}} / r\right)}{\mathrm{d} r}+\frac{(\ell-j)}{a_{\mathrm{s}} r^{2}}\left(\frac{\mathrm{d} \ell}{\mathrm{d} r}-\frac{2 \ell}{r}\right)\right]$.

In order to pass the sonic radius, $r_{\mathrm{s}}$, smoothly, two boundary conditions at $r=r_{\mathrm{s}}$ are given as $\mathcal{D}=\mathcal{N}=0$ at $r=r_{\mathrm{s}}$. For any given $a_{\mathrm{s}, \mathrm{s}}(>0)$ that is the sound speed at the sonic radius, the radial velocity $v_{\mathrm{r}, s}$ at $r=r_{\mathrm{s}}$ is calculated from $\mathcal{D}=0$ as $v_{\mathrm{r}, s}=$ $-a_{\mathrm{s}, \mathrm{s}}[2 \gamma /(\gamma+1)]^{1 / 2}$. From the condition $\mathcal{N}=0$, the angular momentum $\ell_{\mathrm{s}}$ at $r=r_{\mathrm{s}}$ is calculated as $\ell_{\mathrm{s}}=\left[b_{\ell} \pm\left(b_{\ell}^{2}-a_{\ell} c_{\ell}\right)^{1 / 2}\right] / a_{\ell}$, where

$$
\begin{aligned}
a_{\ell}= & 1-\left(\frac{\gamma-1}{\gamma+1}\right)\left\{-2+\frac{1}{\mathcal{D}_{v}}\left[2+\frac{r v_{\mathrm{r}}}{v}\left(1-\frac{2 v_{\mathrm{r}} \tau_{r}}{r}\right)\right]\right\}, \\
b_{\ell}= & \frac{j}{2}\left(\frac{1-\gamma}{1+\gamma}\right)\left\{-\frac{r v_{\mathrm{r}}}{v \mathcal{D}_{v}}\left(1-\frac{2 v_{\mathrm{r}} \tau_{r}}{r}\right)\right. \\
& \left.-2+\frac{1}{\mathcal{D}_{v}}\left[2+\frac{r v_{\mathrm{r}}}{v}\left(1-\frac{2 v_{\mathrm{r}} \tau_{r}}{r}\right)\right]\right\} \\
c_{\ell}= & -\left(\frac{\gamma-1}{\gamma+1}\right) \frac{r v_{\mathrm{r}} j^{2}}{v \mathcal{D}_{v}}\left(1-\frac{2 v_{\mathrm{r}} \tau_{r}}{r}\right)-\Omega_{\mathrm{K}}^{2} r^{4} \\
& -\frac{2 \gamma}{\gamma+1} a_{\mathrm{s}}^{2} r^{3} \frac{\mathrm{d} \ln \left(\Omega_{\mathrm{K}} / r\right)}{\mathrm{d} r}
\end{aligned}
$$

Chakrabarti introduces a very clever method of calculating the value of $\mathrm{d} v_{\mathrm{r}} / \mathrm{d} r$ at $r=r_{\mathrm{s}}$ (e.g. Chakrabarti 1990, 1996). We extend their method to calculate the transonic solutions of the ADAFs with causal viscosity. The value of $\mathrm{d} v_{\mathrm{r}} / \mathrm{d} r$ at $r=r_{\mathrm{s}}$ is calculated by applying L'Hopital's rule as follows. To apply L'Hopital's rule to Eq. (2) at $r=r_{\mathrm{s}}$, we introduce new variables $x=r-r_{\mathrm{s}}, y=v_{\mathrm{r}}-v_{\mathrm{r}, s}, z=\ell-\ell_{\mathrm{s}}$, and $w=a_{\mathrm{s}}-a_{\mathrm{s}, \mathrm{s}}$. The functions $\mathcal{D}$ and $\mathcal{N}$ are expanded near the sonic point as

$\mathcal{D}:\left(\frac{\partial \mathcal{D}}{\partial x}\right)_{\mathrm{s}} x+\left(\frac{\partial \mathcal{D}}{\partial y}\right)_{\mathrm{s}} y+\left(\frac{\partial \mathcal{D}}{\partial z}\right)_{\mathrm{s}} z+\left(\frac{\partial \mathcal{D}}{\partial w}\right)_{\mathrm{s}} w$

$\mathcal{N}:\left(\frac{\partial \mathcal{N}}{\partial x}\right)_{\mathrm{s}} x+\left(\frac{\partial \mathcal{N}}{\partial y}\right)_{\mathrm{s}} y+\left(\frac{\partial \mathcal{N}}{\partial z}\right)_{\mathrm{s}} z+\left(\frac{\partial \mathcal{N}}{\partial w}\right)_{\mathrm{s}} w$

where

$$
\begin{aligned}
\left(\frac{\partial \mathcal{D}}{\partial x}\right)_{\mathrm{s}}= & \left(\frac{\partial \mathcal{D}}{\partial z}\right)_{\mathrm{s}}=0, \quad\left(\frac{\partial \mathcal{D}}{\partial y}\right)_{\mathrm{s}}=2, \quad\left(\frac{\partial \mathcal{D}}{\partial w}\right)_{\mathrm{s}}=2 \sqrt{\frac{2 \gamma}{\gamma+1}} \\
\left(\frac{\partial \mathcal{N}}{\partial x}\right)_{\mathrm{s}}= & \frac{1}{(r-2)^{3}}-\frac{3 \ell^{2}}{r^{4}}-\left(\frac{2 \gamma}{\gamma+1}\right) a_{\mathrm{s}}^{2} \frac{\mathrm{d}^{2} \ln \left(\Omega_{\mathrm{K}} / r\right)}{\mathrm{d} r^{2}} \\
& -\left(\frac{\gamma-1}{\gamma+1}\right) \frac{6 \ell(\ell-j)}{r^{4}} \\
\left(\frac{\partial \mathcal{N}}{\partial y}\right)_{\mathrm{s}}= & 0, \quad\left(\frac{\partial \mathcal{N}}{\partial z}\right)_{\mathrm{s}}=\frac{2 \ell}{r^{3}}+\left(\frac{\gamma-1}{\gamma+1}\right) \frac{2(2 \ell-j)}{r^{3}} \\
\left(\frac{\partial \mathcal{N}}{\partial w}\right)_{\mathrm{s}}= & \left(\frac{-4 \gamma}{\gamma+1} a_{\mathrm{s}} \frac{\mathrm{d} \ln \left(\Omega_{\mathrm{K}} / r\right)}{\mathrm{d} r}\right.
\end{aligned}
$$

Here, $\mathrm{d}^{2} \ln \left(\Omega_{\mathrm{K}} / r\right) / \mathrm{d} r^{2}=\left(5 r^{2}-12 r+12\right) /\left[2 r^{2}(r-2)^{2}\right]$, and $z$ and $w$ are described as $z=(\mathrm{d} \ell / \mathrm{d} r)_{\mathrm{s}} x$ and $w=\left(\partial a_{\mathrm{s}} / \partial r\right)_{\mathrm{s}} x+$ $\left(\partial a_{\mathrm{s}} / \partial v_{\mathrm{r}}\right)_{\mathrm{s}} y$, where

$$
\begin{aligned}
& \left(\frac{\partial a_{\mathrm{s}}}{\partial r}\right)_{\mathrm{s}}=-\left(\frac{\gamma-1}{\gamma+1}\right) \frac{a_{\mathrm{s}}}{v_{\mathrm{r}}} \\
& \left(\frac{\partial a_{\mathrm{s}}}{\partial v_{\mathrm{r}}}\right)_{\mathrm{s}}=\left(\frac{\gamma-1}{\gamma+1}\right)\left\{a_{\mathrm{s}} \frac{\mathrm{d} \ln \left(\Omega_{\mathrm{K}} / r\right)}{\mathrm{d} r}+\frac{(\ell-j)}{a_{\mathrm{s}} r^{2}}\left[\left(\frac{\mathrm{d} \ell}{\mathrm{d} r}\right)_{\mathrm{s}}-\frac{2 \ell}{r}\right]\right\} .
\end{aligned}
$$


Finally, we obtain the quadratic equations of $\mathrm{d} v_{\mathrm{r}} / \mathrm{d} r$ at $r=r_{\mathrm{s}}$ as $\left(\mathrm{d} v_{\mathrm{r}} / \mathrm{d} r\right)_{\mathrm{s}}=a \pm\left(a^{2}-b\right)^{1 / 2}$, which are the solutions of the equation $\left(\mathrm{d} v_{\mathrm{r}} / \mathrm{d} r\right)_{\mathrm{s}}^{2}-2 a\left(\mathrm{~d} v_{\mathrm{r}} / \mathrm{d} r\right)_{\mathrm{s}}+b=0$, where

$$
\begin{aligned}
& a=\frac{\left(\frac{\partial \mathcal{N}}{\partial y}\right)_{\mathrm{s}}+\left(\frac{\partial \mathcal{N}}{\partial w}\right)_{\mathrm{s}}\left(\frac{\partial a_{\mathrm{s}}}{\partial v_{r}}\right)_{\mathrm{s}}-\left(\frac{\partial \mathcal{D}}{\partial x}\right)_{\mathrm{s}}-\left(\frac{\partial \mathcal{D}}{\partial z}\right)_{\mathrm{s}}\left(\frac{\partial \ell}{\partial r}\right)_{\mathrm{s}}-\left(\frac{\partial \mathcal{D}}{\partial w}\right)_{\mathrm{s}}\left(\frac{\partial a_{\mathrm{s}}}{\partial r}\right)_{\mathrm{s}}}{2\left[\left(\frac{\partial \mathcal{D}}{\partial y}\right)_{\mathrm{s}}+\left(\frac{\partial \mathcal{D}}{\partial w}\right)_{\mathrm{s}}\left(\frac{\partial a_{\mathrm{s}}}{\partial v_{\mathrm{r}}}\right)_{\mathrm{s}}\right]}, \\
& b=\frac{-\left(\frac{\partial \mathcal{N}}{\partial x}\right)_{\mathrm{s}}-\left(\frac{\partial \mathcal{N}}{\partial z}\right)_{\mathrm{s}}\left(\frac{\partial \ell}{\partial r}\right)_{\mathrm{s}}-\left(\frac{\partial \mathcal{N}}{\partial w}\right)_{\mathrm{s}}\left(\frac{\partial a_{\mathrm{s}}}{\partial r}\right)_{\mathrm{s}}}{\left(\frac{\partial \mathcal{D}}{\partial y}\right)_{\mathrm{s}}+\left(\frac{\partial \mathcal{D}}{\partial w}\right)_{\mathrm{s}}\left(\frac{\partial a_{\mathrm{s}}}{\partial v_{\mathrm{r}}}\right)_{\mathrm{s}}}
\end{aligned}
$$

In addition to the boundary conditions $\mathcal{D}=\mathcal{N}=0$, when the causal viscosity prescription is adopted, the accretion flows smoothly pass the viscous radius, $r_{v}$, where the condition $\mathcal{D}_{v}=0$ is satisfied. In this case, the two boundary conditions at the viscous radius, $r_{v}$, are given as $\mathcal{D}_{v}=\mathcal{N}_{v}=0$ at $r=r_{v}$. The method presented in this study uses the boundary conditions at the sonic radius, $r_{\mathrm{s}}$, and the viscous radius, $r_{v}$, and does not use the boundary conditions in the outer region $r>r_{v}$ and the inner region $r_{\mathrm{h}}<r<r_{\mathrm{s}}$, where $r_{\mathrm{h}}$ is the radius for the event horizon.

\section{Calculation method and sample solutions}

Based on the formula presented in the previous sections, we can calculate the global transonic solutions for ADAFs with the causal viscosity. The calculation procedures for obtaining the global transonic solutions for ADAFs are as follows:

1. First, we tentatively choose some value of $a_{\mathrm{s}, \mathrm{s}}$ for given values of $r_{\mathrm{s}}$ and $j$ and calculate $v_{\mathrm{r}, s}, \ell_{\mathrm{s}},\left(\mathrm{d} v_{\mathrm{r}} / \mathrm{d} r\right)_{\mathrm{s}},(\mathrm{d} \ell / \mathrm{d} r)_{\mathrm{s}}$, and $\left(\mathrm{d} a_{\mathrm{s}} / \mathrm{d} r\right)_{\mathrm{s}}$ by using Eqs. (7), (10), and (12).

2. Next, we solve the solutions in the range $r_{\mathrm{s}}<r<r_{v}$. In order to do this, we solve Eqs. (7), (10), and (12) from the sonic point to the viscous point by using, e.g., the Runge-Kutta algorithm. Usually, for the initially selected value of $a_{\mathrm{s}, \mathrm{s}}$, the calculated solution does not pass the viscous point where two boundary conditions $\mathcal{D}_{v}=\mathcal{N}_{v}=0$ are satisfied. In such a case, we return to step 1 and again choose the different values of $a_{\mathrm{s}, \mathrm{s}}$ for given values of $r_{\mathrm{s}}$ and $j$. After repeating these procedures, we can determine the value $a_{\mathrm{s}, \mathrm{s}}$, which gives the solution satisfying the boundary conditions $\mathcal{D}=\mathcal{N}=0$ at $r=r_{\mathrm{s}}$ and $\mathcal{D}_{v}=\mathcal{N}_{v}=0$ at $r=r_{v}$.

3. After solving the solutions in $r_{\mathrm{s}}<r<r_{v}$, we solve Eqs. (7), (10), and (12) in the range $r_{v}<r$ by using the values of $a_{\mathrm{s}, \mathrm{s}}$ for given values of $r_{\mathrm{s}}$ and $j$ by using, e.g., the Runge-Kutta algorithm.

4. Finally, we solve Eqs. (7), (10), and (12) in the range $r_{\mathrm{h}}<$ $r<r_{\mathrm{s}}$ by using the values of $a_{\mathrm{s}, \mathrm{s}}$ for given values of $r_{\mathrm{s}}$ and $j$ by using, e.g., the Runge-Kutta algorithm.

The third and the fourth steps can be interchanged. By this procedure, the transonic solutions are obtained for given values of $r_{\mathrm{s}}$ and $j$. At the third step, the physical values, such as $\mathrm{d} \ell / \mathrm{d} r$, at the viscous radius $r=r_{v}$ are used if necessary to obtain the solutions. Usually, the numerical calculation of the coupled equations are naturally performed through the viscous point, and the calculations in the third steps are successively done after passing through the viscous radius. However, in some cases, the numerical calculation is stopped at the viscous radius. In such cases, we evaluate the physical values at the viscous point by using L'Hopital's rule, which give the value $\mathrm{d} \ell / \mathrm{d} r$ at the viscous radius $r=r_{v}$, and then we calculate the coupled equations for the global solution from the viscous radius to the outward direction $r>r_{v}$. In the same way as the calculation of $\left(\mathrm{d} v_{\mathrm{r}} / \mathrm{d} r\right)_{\mathrm{s}}$ as described in the last section, the value $(\mathrm{d} \ell / \mathrm{d} r)_{r=r_{v}}$ is calculated as $(\mathrm{d} \ell / \mathrm{d} r)_{r=r_{v}}=a_{v} \pm\left(a_{v}^{2}-b_{v}\right)^{1 / 2}$, where

$$
\begin{aligned}
a_{v}= & \frac{1}{2}\left\{\left(\frac{\partial \mathcal{N}_{v}}{\partial z}\right)_{v}+\left(\frac{\partial \mathcal{N}_{v}}{\partial w}\right)_{v}\left(\frac{\partial a_{\mathrm{s}}}{\partial \ell}\right)_{v}-\left(\frac{\partial a_{\mathrm{s}}}{\partial r}\right)_{v}-\left(\frac{\partial \mathcal{D}_{v}}{\partial x}\right)_{v}\right. \\
& \left.-\left[\left(\frac{\partial \mathcal{D}_{v}}{\partial y}\right)_{v}+\left(\frac{\partial \mathcal{D}_{v}}{\partial w}\right)_{v}\left(\frac{\partial a_{\mathrm{s}}}{\partial v_{\mathrm{r}}}\right)_{v}\right] \frac{\mathcal{N}_{v}}{\mathcal{D}_{v}}\right\} \\
& /\left[\left(\frac{\partial \mathcal{D}_{v}}{\partial z}\right)_{v}+\left(\frac{\partial \mathcal{D}_{v}}{\partial w}\right)_{v}\left(\frac{\partial a_{\mathrm{s}}}{\partial \ell}\right)_{v}\right] \\
b_{v}= & -\left\{\left(\frac{\partial \mathcal{N}_{v}}{\partial x}\right)_{v}+\left(\frac{\partial \mathcal{N}_{v}}{\partial w}\right)_{v}\left(\frac{\partial a_{\mathrm{s}}}{\partial r}\right)_{v}\right. \\
& \left.+\left[\left(\frac{\partial \mathcal{N}_{v}}{\partial y}\right)_{v}+\left(\frac{\partial \mathcal{N}_{v}}{\partial w}\right)_{v}\left(\frac{\partial a_{\mathrm{s}}}{\partial v_{\mathrm{r}}}\right)_{v}\right] \frac{\mathcal{N}_{v}}{\mathcal{D}_{v}}\right\} \\
& /\left[\left(\frac{\partial \mathcal{D}_{v}}{\partial z}\right)_{v}+\left(\frac{\partial \mathcal{D}_{v}}{\partial w}\right)_{v}\left(\frac{\partial a_{\mathrm{s}}}{\partial \ell}\right)_{v}\right]
\end{aligned}
$$

Here,

$$
\begin{aligned}
& \left(\frac{\partial \mathcal{D}_{v}}{\partial x}\right)_{v}=\left(\frac{\partial \mathcal{D}_{v}}{\partial z}\right)_{v}=0, \quad\left(\frac{\partial \mathcal{D}_{v}}{\partial y}\right)_{v}=\frac{2}{\alpha^{1 / 2} a_{\mathrm{s}}}, \quad\left(\frac{\partial \mathcal{D}_{v}}{\partial w}\right)_{v}=\frac{2}{a_{\mathrm{s}}} \\
& \left(\frac{\partial \mathcal{N}_{v}}{\partial x}\right)_{v}=-\frac{\mathcal{N}_{v}}{r}-\frac{(\ell-j)}{r v_{\mathrm{r}}} \frac{\mathrm{d}\left(\Omega_{\mathrm{K}} r\right)}{\mathrm{d} r} \\
& \left(\frac{\partial \mathcal{N}_{v}}{\partial y}\right)_{v}=\frac{(\ell-j)}{r v_{\mathrm{r}}}\left[\frac{v_{\mathrm{r}}\left(\Omega_{\mathrm{K}} r-2 v_{\mathrm{r}}\right)}{\alpha a_{\mathrm{s}}^{2}}-2\right] \\
& \left(\frac{\partial \mathcal{N}_{v}}{\partial z}\right)_{v}=\frac{1}{r}\left[\frac{v_{\mathrm{r}}\left(\Omega_{\mathrm{K}} r-2 v_{\mathrm{r}}\right)}{\alpha a_{\mathrm{s}}^{2}}+2\right] \\
& \left(\frac{\partial \mathcal{N}_{v}}{\partial w}\right)_{v}=\frac{-2(\ell-j)\left(\Omega_{\mathrm{K}} r-2 v_{\mathrm{r}}\right)}{r v_{\mathrm{r}} a_{\mathrm{s}}}, \\
& \left(\frac{\partial a_{\mathrm{s}}}{\partial r}\right)_{=}=\left(\frac{\gamma-1}{\gamma+1}\right)\left[a_{\mathrm{s}} \frac{\mathrm{d} \ln \left(\Omega_{\mathrm{K}} / r\right)}{\mathrm{d} r}-\frac{2 \ell(\ell-j)}{a_{\mathrm{s}} r^{3}}\right] \\
& \left(\frac{\partial a_{\mathrm{s}}}{\partial v_{\mathrm{r}}}\right)=-\frac{a_{\mathrm{s}}}{v_{\mathrm{r}}}\left(\frac{\gamma-1}{\gamma+1}\right), \quad\left(\frac{\partial a_{\mathrm{s}}}{\partial \ell}\right)=\left(\frac{\gamma-1}{\gamma+1}\right) \frac{(\ell-j)}{a_{\mathrm{s}} r^{2}}
\end{aligned}
$$

where $\mathrm{d}\left(\Omega_{\mathrm{K}} r\right) / \mathrm{d} r=-\Omega_{\mathrm{K}}(r+2) /[2(r-2)]$. The values of $v_{\mathrm{r}}, \ell$ and $a_{\mathrm{S}}$ at the viscous radius are determined to satisfy $\mathcal{D}_{v}=\mathcal{N}_{v}=0$.

It is noted that, by using the method described above, we do not use the outer boundary conditions for $r>r_{v}$ and the inner boundary conditions for $r<r_{\mathrm{s}}$. If we want the solutions for specified outer or inner boundary conditions, we should choose the values of $r_{\mathrm{s}}$ and $j$ to satisfy the specified outer or inner boundary conditions. The correspondences between the values $r_{\mathrm{s}}$ and $j$ and the outer solutions are investigated by Lu et al. (1999) for the special solutions satisfying the condition $\ell=j$ at $r=r_{\mathrm{s}}$, and they provide solutions for the ADAF-thick disk solution, the ADAF-thin disk solution, and the alpha-type solution connecting the inner of outer regions. The special condition $\ell=j$ at $r=r_{\mathrm{s}}$ simplify the equations described in the last section. In the case of the solutions including the cases for $\ell \neq j$ at $r=r_{\mathrm{s}}$, which can be calculated by the method described above, we expect the basic picture to be same as the solutions with $\ell=j$ at $r=r_{\mathrm{s}}$ in Lu et al. (1999).

In Fig. 1, we present the sample solutions for ADAF-thick disk and ADAF-thin disk for $\alpha=0.1$ and $\gamma=1.5$; the radial velocity $v_{\mathrm{r}}$ (top-left panel), the angular momentum (top-right panel), the sound speed $a_{\mathrm{s}}$ (bottom-left panel), and H/r (bottomright panel). It is also shows the positions of the sonic radius, $r_{\mathrm{s}}$, and the viscous radius, $r_{v}$ for all panels. We give the solutions in the range $r_{\mathrm{h}}<r<10^{1.5}$, and the adopted values of $\log r_{\mathrm{s}}$ and $j$ are $\log r_{\mathrm{s}}=0.7$ and $j=2.0$. Here, we adopt the 

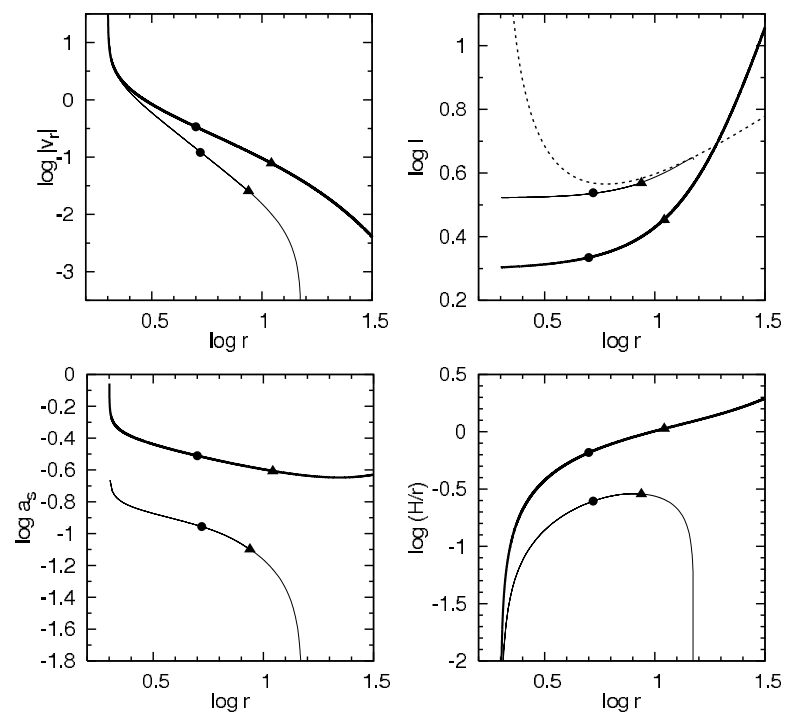

Fig. 1. The sample solutions for ADAF-thick disk (thick lines) and ADAF-thin disk (thin lines) by solid lines for $\alpha=0.1$ and $\gamma=1.5$; the radial velocity $v_{\mathrm{r}}$ (top-left panel), the angular momentum (top-right panel), the sound speed $a_{\mathrm{s}}$ (bottom panel) and $H / r$ (bottom-right panel). The filled circles and triangles denote the positions of the sonic radius, $r_{\mathrm{s}}$, and the viscous radius, $r_{v}$. In the panel of $\log \ell$, we also plot the line of the Keplerian angular momentum $\ell_{\mathrm{K}}\left(=\Omega_{\mathrm{K}} r^{2}\right)$ by a dotted line.

Runge-Kutta-Fehlberg algorithm for numerical calculations of the coupled differential equations of $v_{\mathrm{r}}, \ell$ and $a_{\mathrm{s}}$. The line of $\log \left|v_{\mathrm{r}}\right|$ crosses the line of $\log [2 \gamma /(\gamma+1)]^{1 / 2} a_{\mathrm{s}}$ at the sonic radius, $r_{\mathrm{s}}$, and the line of $\log c_{v}$ at the viscous radius, $r_{v}$, respectively. In the outer region $r>r_{v}$, the angular momentum of the solution achieves the Keplerian value. According to the complete parameter search of the ADAF solutions by Lu et al. (1999), the ADAF solutions are classified into the ADAF-thick disk solution, the ADAF-thin disk solution, and the $\alpha$-type solution connecting the inner region or the outer region. While the parameter spaces of $r_{\mathrm{s}}$ and $j$ for the ADAF-thick solutions and the $\alpha$-type solutions are relatively wide, which can be easily confirmed numerically, the parameter spaces for the ADAF-thin disk solutions are quite limited. By the calculation procedures presented in this paper, we can completely cover the possible parameter spaces of the transonic solutions of ADAFs.

\section{Discussion}

The formula in the present study can become the formula for the acausal viscous prescription if we set the diffusion timescale $\tau_{v}$ to null. As a result, the analytic expansion around the singular points presented in this paper are also used to calculate the global solution for ADAF with the acausal viscosity. In this case, since the boundary condition at the viscous point cannot be used, the boundary condition at the other radius is required, e.g. no-torque condition at the horizon. Although the finite diffusion velocity is physically motivated, the resulting solutions have generally nonzero torque at the horizon, which seems to be incorrect from the point of view of the relativity. Also, in the causal viscous prescription, the differential equation for the angular momentum becomes more complex than that of the acausal viscosity. Although the finite diffusion speed is more reasonable than the infinite diffusion speed, it is noted that the calculations of the ADAF structure by using the causal viscous prescription may not be clearly better than the calculations using the acausal viscosity.
In the fully general relativistic calculations performed by Gammie \& Popham (1998), which use the causal viscosity prescription, the boundary condition at the viscous point is used when calculating the global solution. In Eq. (60) of Gammie \& Popham (1998), although the viscous stress tensor at the horizon is finite if measured in the local rest frame, i.e. $t_{(r)(\phi)} \neq 0$, the viscous stress tensor at the horizon becomes null when measured in the Boyer-Lindquist frame, i.e. $t^{r}{ }_{\phi}=0$, because of $\mathcal{D}=0$ at the horizon. Since the location of the horizon is not the special location from the point of view of the local observer moving along the fluid's motion, non-zero stress in the local rest frame seems to be quite natural. The zero-torque condition arises only when we use the coordinate system with the coordinate singularity at the horizon, such as the Boyer-Lindquist coordinate, and then measure the viscous stress in these frames. Then, the problem of the non-zero torque or zero-torque at the horizon are the issues that require the fully relativistic treatments with the spacetime structure and the frame-transformation between the observers measuring the viscous stress. Thus, it may be impossible to clearly resolve the torque problem at the horizon by using the calculations with the pseudo-Newtonian potential as in the present study.

\section{Concluding remarks}

We have presented the basic equations and sample solutions for the steady-state global solutions of the ADAFs using the causal viscous prescription and the Paczyńskii-Wiita potential by means of the algorithm of explicit numerical integrations, such as the Runge-Kutta method. We also presented the calculation procedures for obtaining these solutions. In this calculation procedure, we first solved the physical values at the sonic radius where L'Hopital's rule is used. The method presented in this paper enables us to stably solve the global solutions of ADAFs by the Runge-Kutta method, and the all parameter spaces of $r_{\mathrm{s}}$ and $j$ for the transonic solutions of ADAFs can be covered in this method. If we set the diffusion timescale to null, the formalism in this study includes the case of the acausal viscosity, which is usually used in past studies. Since the calculation methods in this study use the analytic expansion around the singular point and the numerical integration is performed by the explicit integration, the numerical calculations are faster.

Acknowledgements. The author is grateful to S. Mineshige and K. Watarai for useful discussion. This research was partially supported by the Ministry of Education, Culture, Sports, Science and Technology, Grant-in-Aid for Japan Society for the Promotion of Science (JSPS) Fellows (17010519).

\section{References}

Abramowicz, M. A., \& Kato, S. 1989, ApJ, 336, 304

Becker, P. A., \& Le, T. 2003, ApJ, 588, 408

Chakrabarti, S. K. 1990, Theory of Transonic Astrophysical Flows (Singapore: World Scientific)

Acrobat, S. K. 1996, ApJ, 464, 664

Gammie, C. F., \& Popham, R. 1998, ApJ, 498, 313

Kato, S., Fukue, J., \& Mineshige, S. 1998, Black-Hole Accretion Disks (Kyoto: Kyoto Univ. Press)

Lu, J.-F., Gu, W.-M., \& Yuan, F. 1999, ApJ, 523, 340

Narayan, R., Kato, S., \& Honma, F. 1997, ApJ, 476, 49

Paczyński, B., \& Wiita, P. 1980, A\&A, 88, 23

Papaloizou, J. C. B., \& Szuszkiewicz, E. 1994, MNRAS, 268, 29 